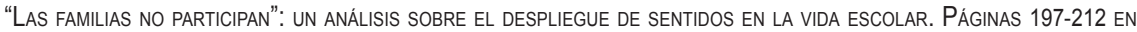
Revista de la Escuela de Ciencias de la Educación, año 10, número 9, enero a diciembre de 2014. ISSN 1851-6297. ISSN EN LINEA 2362-3349.

\title{
"LAS FAMILIAS NO PARTICIPAN": UN ANÁLISIS SOBRE EL DESPLIEGUE DE SENTIDOS EN LA VIDA ESCOLAR
}

\author{
Por Carolina Scavino (Universidad de Buenos Aires)*, Argentina. \\ cscavino@gmail.com
}

Recibido: 04/07/2014 Aceptado: 01/08/2014

\section{Resumen}

Las relaciones entre familias y escuelas han sido analizadas desde diferentes puntos de vista, dando como resultado una literatura específica con recomendaciones y consideraciones a tener en cuenta para que dichos vínculos lleguen a buen puerto en la común tarea de "educar" a los niños/as.

En este campo de producción, este trabajo ofrece un análisis sobre los sentidos que se encubren tras el malestar que se manifiesta en la enunciación de la no participación de las familias en la escuela en la vida escolar.

A partir de un estudio de caso sobre los procesos de participación de las familias en la escuela, realizado en un establecimiento educativo de nivel primario de la zona sur de la Ciudad Autónoma de Buenos Aires, se analiza la función que cumple la expresión "las familias no participan" como representación social en la trama de interacciones establecidas entre ambas instituciones.

De este modo, el trabajo se propone explicitar ciertos conflictos que regulan los procesos de interacción entre familia y escuela, y evidenciar el poder simbólico que está en juego en los intercambios, alimentando el sentido de "individuación" en torno al cual se ha construido la práctica escolar.

\section{Palabras Clave}

Familias - Escuela - Procesos de participación - Nivel primario - Estudio de caso.

\section{Abstract}

Relationships between families and schools have been analyzed from different points of view, resulting in a specific literature with recommendations and considerations

* Lic. en Psicología (UBA), Mgtr. En Psicología Educacional (UBA), Doctora con mención en Ciencias Sociales y Humanas (UNQ). Docente-Investigadora en la Facultad de DerechoUBA y en la Universidad Nacional General Sarmiento (UNGS). 
Revista de la Escuela de Ciencias de la Educación, año 10, número 9, enero a diciembre de 2014. Páginas 197-212. ISSN 1851-6297. ISSN EN LINEA 2362-3349. "LAS FAMILIAS NO PARTICIPAN": UN ANÁLISIS SOBRE EL DESPLIEGUE DE SENTIDOS en la Vida escolar. Carolina Scavino.

to take into account that those links come to fruition in the common task of "educating" children.

In this field of production, this paper provides an analysis of the meanings that are concealed behind the unrest manifested in the statement of non-participation of families in the school's school life.

From a case study on the processes of family involvement in school, performed in a primary educational establishment in the south of Buenos Aires, the role of the expression is parsed "families not participate" as social representation in the web of interactions established between the two institutions.

Thus, the paper proposes explicit certain conflicts that regulate the processes of interaction between family and school, and to demonstrate the symbolic power is at stake in trade, fueling the sense of "individuation" around which has been built school practice.

\section{Key Words}

Families - School - Participation process - Primary level - Case study.

\section{Introducción}

Las relaciones de la familia con la escuela han sido abordadas ampliamente por la literatura académica y de divulgación. De modo sucinto, podría decirse que el tema ha sido vinculado con la promoción del aprendizaje escolar y estrategias alternativas ante el fracaso escolar o bien con formas de trabajo conjunto respecto a la transmisión de normas sociales y modos de organizar las actividades educativas destinadas de los niños/as en desarrollo (Scavino, 2012). En esta diversidad de temáticas, es oportuno aclarar el punto de vista desde el cual este trabajo aborda dichas relaciones.

Se parte de la idea que la "escuela" como la "familia" son prácticas sociales modernas, emergentes de la consolidación de los Estados-Nación y categorías de pensamiento, genéricas y normativas, que soslayan formas singulares en que se reproducen dichas prácticas.

El reconocimiento de esta distinción, también supone identificar el punto de encuentro en el cual familia y escuela establecen su vínculo. Dicho punto se da en la concepción de "educar a las nuevas generaciones". Una tarea compartida en una sociedad organizada en torno a la idea de la división del trabajo y la diferenciación de los espacios sociales -públicos y privados-, lo cual supone diferenciar roles y funciones vinculado a la tarea en común (Herztfeld, 1992, Scavino, 2007, 2012)

De este modo, las familias y las escuelas son convenciones "naturalizadas", instituciones sociales (Douglas, 1996) que no remiten simplemente a una definición sino a todo un conjunto de conceptos, concepciones, que sustentan su existencia. 
Revista de la Escuela de Ciencias de la Educación, año 10, número 9, enero a diciembre de 2014. Páginas 197-212. ISSN 1851-6297. ISSN EN LINEA 2362-3349. "LAS FAMILIAS NO PARTICIPAN": UN ANÁLISIS SOBRE EL DESPLIEGUE DE SENTIDOS en la vida escolar. Carolina Scavino.

Por otra parte, el análisis de dichas relaciones se complejiza si se comprende que la regulación jurídica y los efectos en la constitución subjetiva de dichas prácticas no son las mismas. Por ejemplo al interior de las prácticas familiares, los miembros crecen y se desarrollan, forjan su existencia; en cambio, en las prácticas escolares, éstos despliegan su existencia, las ponen en juego y reciben marcas que orientan su rumbo (1) (Carcova, 1998; Bourdieu, 2007; Scavino, 2011; 2012; Chardon, 2011).

Tomando como punto de partida estas consideraciones, a continuación se analiza una "queja escolar" que fue identificada en la realización de un estudio de caso que se propuso indagar los procesos de participación de las familias en la escuela durante los primeros años de escolaridad primaria en contextos de pobreza urbana, en un establecimiento educativo de gestión pública y jornada simple de la zona sur de la Ciudad Autónoma de Buenos Aires (2). Su hallazgo consiste en haber podido explicitar y evidenciar las regulaciones del poder que se ciernen bajo el modo de una queja, a beneficio de sostener ciertos pilares ideológicos que dieron origen a las prácticas escolares.

\section{"Las familias no participan": algunas consideraciones teóricas}

La queja escolar sobre "las familias no participan" no es una novedad para aquellos que se dedican al tema. Diferentes estudios han identificado que la frase refiere a una falta de respuesta por parte de las familias ante ciertas demandas escolares, que por lo general son de carácter subsidiario a los objetivos escolares que se fijan como metas (Cerletti, 2006; Santillan, 2008a; Bravin, 2004).

Al mismo tiempo, las investigaciones también han demostrado que las familias tienen un rol activo y participativo en la escolarización de sus hijos/as que no es visibilizado por los agentes de la instituciones escolares (Santillan y Cerletti, 2011; Cerletti, 2010; Santillan, 2007, 2008b; Achilli, 2000, 2010; Neufeld, 2000).

De este modo, los antecedentes presentan un panorama complejo y "aparentemente" contradictorio, respecto a las formas que se tienden a percibir los procesos participativos entre familia y escuela.

El estudio realizado confirmó los datos y se propuso entonces avanzar en el análisis de dicha contradicción. La expresión "las familias no participan" es una frase muy frecuente y cotidiana, que se manifiesta en el contexto escolar tanto por diversos agentes educativos, como también puede ser registrada por parte de algunos padres que integran algún órgano de gestión vinculado al establecimiento, como por ejemplo las cooperadoras escolares.

Así, por un lado, la frase puede ser concebida como un producto cultural, una expresión común en el escenario escolar que es importante interpretar. Desde una perspectiva formal del lenguaje, la frase puede ser tomada como una descripción de un hecho real y objetivo que acontece en un contexto particular; mientras que si se interpreta desde una perspectiva sociocultural, ella 
Revista de la Escuela de Ciencias de la Educación, año 10, número 9, enero a diciembre de 2014. Páginas 197-212. ISSN 1851-6297. ISSN EN LINEA 2362-3349. "LAS FAMILIAS NO PARTICIPAN": UN ANÁLISIS SOBRE EL DESPLIEGUE DE SENTIDOS en la vida escolar. Carolina Scavino.

informa no tanto la realidad como el juego de intereses (3) que están regulando en ese escenario social (Duranti, 2000; Bruner, 2003; Bourdieu, 2007). En este sentido, para la investigación la frase ha constituido un elemento que revela la dimensión cultural de las relaciones entre la familia y la escuela, y advierte sobre las relaciones de poder que regulan el proceso de la vida social (Rockwell, 2009; Guber, 2004; Bourdieu, 2007).

Finalmente, por otro lado, la frecuencia con la que se recurre a la misma, da cuenta del carácter naturalizado y su eficacia en el modo de regular las interacciones entre familias y escuela. Si se toma en consideración el señalamiento de Jodelet (1998) respecto a que una representación es una reconstrucción de la realidad, lograda a partir del lenguaje en la interacción con los otros y los objetos del mundo. La frase en cuestión, no es más que un emergente de la vida cotidiana en las escuelas. Es una representación social, en tanto producción simbólica originada en las formas de participación de los agentes en la vida diaria, y susceptibles de modificarse/transformarse según las interacciones establecidas con los "otros", es decir reconociendo en la alteridad los motores de la transformación de las representaciones (Jovchelovitch, 2007; Markova, 2006).

Si se considera entonces la frase como un emergente de la cultura escolar, se advierte que es en la intersubjetividad y el reconocimiento de la alteridad, donde se gestan las posibles transformaciones de los saberes culturales que allí se crean.

La cultura escolar no es estática a lo largo del tiempo y se han identificado diversos planos que la conforman (Rockwell, 2010): un plano de procesos de larga duración, que refiere a elementos culturales que se mantienen en el tiempo y están presentes en la cotidianidad escolar; de continuidades relativas, donde se advierten que contenidos de diferentes tiempos históricos se entrecruzan $\mathrm{y}$ articulan estableciendo en el tiempo presente un juego de continuidades y discontinuidades y uno de co-construcción que hace referencia a los encuentros en la escuela, a las interacciones y el trabajo con los objetos culturales en el tiempo presente.

Interpretar las relaciones de la familia y la escuela, en términos de los intercambios e interacciones que allí se producen y el lugar del reconocimiento entre ambas partes, permite develar la lucha política que dinamiza la vida social.

En esta oportunidad, este trabajo presenta un análisis sobre los sentidos que circulan en torno a la frase "las familias no participan" y lo que perdura en el tiempo a pesar de los cambios que se promueven.

\section{“Las familias no participan": un análisis sobre el despliegue de sentidos que ella denuncia en la vida escolar.}

"Las familias no participan", "no están" o "no vienen" es el modo de justificar ciertas formas que adopta la dinámica escolar, ciertas intenciones de los agentes escolares y un modo de entablar vínculos con las familias. 
Revista de la Escuela de Ciencias de la Edducación, año 10, número 9, enero a diciembre de 2014. Páginas $197-212$. ISSN 1851-6297. ISSN EN LINEA 2362-3349. "LAS FAMILIAS NO PARTICIPAN": UN ANÁLISIS SOBRE EL DESPLIEGUE DE SENTIDOS en la vida escolar. Carolina Scavino.

Recuerdo el momento en que inicié el trabajo de campo de mi investigación y fui invitada a una fiesta folklórica. Ese día tuve la oportunidad de conocer a gran parte del equipo docente de la institución y les comenté que tenía interés en estudiar las relaciones de la familia y la escuela. Los docentes se rieron y con cierta ironía me aconsejaron que me pusiera a escribir la tesis porque "no hay relación". Ésta sólo fue la "carta de presentación" de una referencia recurrente en el avance de la investigación.

La frase fue identificada no solo en el cuerpo docente, sino en los auxiliares y miembros de la cooperadora. En todos los casos, su referencia justificaba las dificultades que tenían en cumplir sus objetivos escolares y la frustración que esto les ocasionaba, y a medida que se avanzaba en los registros, la frase presentó un carácter cada vez más naturalizado en su denuncia.

A su vez, se pudo registrar que su presencia en el discurso escolar no correspondía a un emergente actual, si bien tenía cierto tiempo de existencia, la frase "las familias no participan" estaba relacionada a cierto momento histórico de transformación de la cultura escolar, antes del cual, los vínculos con las familias no eran vividos negativamente (4); sin embargo, en la actualidad la frase era un hecho "evidente", que a veces se asumía con resignación y a veces con la esperanza de dar en algún momento con un grupo de padres que "pueda comprender lo que necesitan los niños" (Scavino, 2012).

Sus efectos no solo regulaban la vida cotidiana de la escuela, sino también en el trabajo de la propia investigación. Su referencia, cerraba sentido en los diálogos que se promovían en el marco de las entrevistas con los agentes escolares, o bien despertaba en los entrevistados, iniciativas por probar nuevas acciones "concretas" con la esperanza de lograr la "deseada" (5) participación.

A partir de estas reacciones, se decidió en la investigación ajustar las estrategias de indagación. Por un lado, se colaboró con la escuela en la puesta en marcha de acciones orientadas a promover nuevas estrategias de participación con la familia, con el objetivo de analizar el modo en que las desarrollaban y gestionaban; y por otro, se buscó ampliar el diálogo sobre las relaciones de la familia y la escuela hacia ámbitos extra-escolares y analizar el nivel de recurrencia de la frase en otros contextos socio-comunitarios abocados y/o vinculados con tareas educativas (Scavino, 2012).

A continuación se expone el resultado obtenido a partir de la estrategia de investigación asumida.

\section{- "Las familias no participan" desde el punto de vista docente.}

En una de las entrevistas al director de la escuela, éste me comenta que un rasgo que distinguía a esta escuela de otras es que "(en las escuela) donde hay una contención mayor por parte de las familias en cuanto a los chicos, hay como una merma de tareas (para la escuela)", planteaba que contar con "mayor contención" por parte de las familias evitaba tener una escuela que actúe como "bomberos, (...) apagando el fuego en todos los rincones". 
Revista de la Escuela de Ciencias de la Educación, año 10, número 9, enero a diciembre de 2014. Páginas 197-212. ISSN 1851-6297. ISSN EN LINEA 2362-3349. "LAS FAMILIAS NO PARTICIPAN": UN ANÁLISIS SOBRE EL DESPLIEGUE DE SENTIDOS en la Vida escolar. Carolina Scavino.

En entrevistas con docentes de grado, se pudo registrar que la escuela reconoce que adopta una posición prescriptiva respecto al trabajo con las familias que debiera ser evitado, aunque señalan que es necesario por el tipo de población con la que trabajan, "hay que enseñar a ser padres", justificado en que hay acciones familiares que son disfuncionales a las lógicas, dinámicas y objetivos que la escuela se propone. Es decir, en el reclamo sobre la falta de participación de las familias en la escuela se denuncia una situación de emergencia en la que se desarrolla la actividad escolar por las problemáticas que deben afrontar a la hora de cumplir con sus objetivos.

La frase adquiere entonces un sentido de "queja", de expresión de un malestar, como camino alternativo por el que los agentes escolares intentan paliar la adversidad en la que desarrollan su tarea (6).

En esta misma línea, en una de las entrevistas al equipo de orientación escolar (EOE) se identificó como malestar que las familias "no organizan la lógica migratoria en función al calendario escolar", en referencia a las ausencias de los niños/as y sus interrupciones en el trabajo escolar; o en las entrevistas al personal auxiliar, expresaban su malestar por las diferencias entre la organización de hábitos y costumbres familiares en comparación a las que eran necesarias para habitar y mantener la escuela.

Respecto al análisis de las acciones que propuso la escuela, para promover la participación de las familias en el período en que se desarrollo el trabajo de investigación, se recupera una nota elaborada por un docente, en relación con algunos consejos para acompañar la escolaridad de los chicos/as del primer ciclo -1ero., 2do. y 3er. Grado- y que fue entregado a las familias en el marco de las reuniones de padres:

NADIE PUEDE SOLO, LOS CHICOS NECESITAN DE SUS FAMILIAS, LAS FAMILIAS DE LA ESCUELA, Y NOSOTROS COMO ESCUELA TAMBIÉN NECESITAMOS DE UDS LOS PADRES.

- Es fundamental un buen descanso para sostener la atención necesaria en el trabajo escolar. Evitar las trasnochadas les permite a los niños reparar energías para la mañana siguiente.

- Es bueno organizar una rutina diaria y sostenerla, poder hacer la tarea luego de almorzar les permite terminar a tiempo con lo pedido y luego disponer de un momento para el juego. De este modo como padres también estamos trabajando la idea de la responsabilidad que nuestros mismos niños deben tener para con la escuela.

- Es muy importante que todos los días miren todos sus cuadernos. Con esto hacemos dos cosas: nos enteramos que cosas están aprendiendo y en que necesitan ser ayudados. 
Revista de la Escuela de Ciencias de la Educación, año 10, número 9, enero a diciembre de 2014. Páginas 197-212. ISSN 1851-6297. ISSN EN LINEA 2362-3349. "LAS FAMILIAS NO PARTICIPAN": UN ANÁLISIS SOBRE EL DESPLIEGUE DE SENTIDOS en la vida escolar. Carolina Scavino.

- Entusiasmarlos para que lean y escriban. Compartir diferentes situaciones de lectura; recetas, libros, chistes o el mismo cuaderno de clases. Leer en voz alta o simplemente leer imágenes de libros, carteles, etc. Escribir juntos listas, números, notas.

- Participar en las convocatorias de la escuela, reuniones, actos, clases abiertas, etc. De esta forma como padres están atentos a las propuestas de la institución y valoran el trabajo de sus hijos y docentes a cargo.

- Asistir a reuniones de padres, ya que es un momento y espacio para intercambiar ideas acerca de cómo ayudar a sus hijos y como colaborar en los aprendizajes

- Alentarlos permanentemente, reconocer sus avances, ayudarlos a resolver cuando sienten que no pueden, invitarlos a mejorar, reconocer en ellos los esfuerzos.

- Enseñarles a que preguntar es importante ya que equivocarse no es un error sino la posibilidad de aprender

- Otras propuestas:

La nota evidencia el carácter prescriptivo-normativo del discurso escolar que opera sobre las familias y podría tensionar su interacción, al no reconocer que las familias también gozan de autoridad en el estilo de las funciones educativas que tienen a su cargo. La nota, sin proponérselo, establece estándares sobre "lo correcto" o "incorrecto" y si bien da lugar a que los padres puedan elaborar otras propuestas, no se sugiere la revisión y/o discusión de las anteriormente mencionadas.

Finalmente, a partir de un análisis sobre las formas de organización de la tarea escolar: ingreso y egresos, recreos, tiempos de trabajo en el aula, etc. se pudo explicitar que las familias no forman parte del vínculo pedagógico. Esto puede ser una afirmación que no necesita ser registrada por la investigación para ser asumida, sin embargo su explicitación permite aclarar los sentidos de la "no participación de las familias". Ellas son "invitadas" a la escuela, la bordean, tienen restricciones para circular por su interior y como veremos en el apartado siguiente, es una restricción respetada y aceptada por las familias sobre cómo "deben ser las cosas" en la escolarización de sus hijos/as.

Las familias acompañan, llevan y traen, están presentes en los alrededores de la escuela. El trabajo docente, delimita la circulación de las familias y produce un efecto de subjetivación, de "individuación" en los niños/as.

\section{- "Las familias no participan" desde el punto de vista de las familias.}

A partir de trabajo realizado con las familias se pudo observar que si bien no comparten tan claramente la idea de que "las familias no participan", si 
Revista de la Escuela de Ciencias de la Educación, año 10, número 9, enero a diciembre de 2014. Páginas 197-212. ISSN 1851-6297. ISSN EN LINEA 2362-3349. "LAS FAMILIAS NO PARTICIPAN": UN ANÁLISIS SOBRE EL DESPLIEGUE DE SENTIDOS en la Vida escolar. Carolina Scavino.

acuerdan con el malestar que plantea la escuela al enunciar la frase cuando está en relación a la falta de acompañamiento de las familias en el cumplimiento de las tradicionales tareas escolares.

En las entrevistas realizadas, se pudo identificar una distinción de sentido respecto a "las familias no participan". Por un lado, avalan y justifican la no participación de la familia, cuando los problemas que la escuela les plantea están en relación a la convivencia en la escuela, el comportamiento de los hijos/as dentro del establecimiento, faltas al docente, el no aprendizaje de los contenidos, etc.; consideran que éstos no son temas sobre los que "ellas", como familias, puedan avanzar porque no están en la escuela, no forman parte de ese momento en que se producen los "conflictos".

Frente a estas demandas escolares su no participación refiere a "no saber qué hacer", los hechos que les plantean exceden sus capacidades y toma de decisiones.

Por otro lado, comparten y avalan la responsabilidad que tienen como familia en acompañar la escolarización de los niños/as, en estos casos, muchas familias reconocen tener dificultades por su nivel educativo para hacer frente al apoyo en las tareas escolares, sin embargo estas dificultades no las paralizan ni las dejan sin resolver, desarrollan diversas estrategias familiares según los recursos disponibles, como solicitar el acompañamiento de los hermanos mayores, primos/as u otros parientes o bien recurrir a algún centro comunitario del barrio que brinde apoyo escolar.

Estas estrategias básicamente se basan en una delegación de las tareas de modo práctico (7), es decir sin ser consciente de modo acabado de lo que están solicitando con su accionar.

Para gran parte de las familias, enviar a sus hijos/as al apoyo escolar implica ceder sus funciones y responsabilidades en otros agentes educativos. Los padres asumen que los referentes cumplirán las tareas que ellos no se consideran a la altura, aunque esto no se conversa de modo explícito por cada una de las partes, como tampoco se aclara que un apoyo no es sólo realizar una tarea y a su vez, los padres al delegar, no ejercen tareas de supervisión sobre lo que se hace en los apoyos, considerando que si a pesar de ir a los apoyos el hijo/a no progresa, "algo tiene el hijo/a".

Estas estrategias de ayuda que emprenden los padres para hacer frente a los problemas que visualizan en la escolarización de sus hijos/as, son formas de participación y acompañamiento a los mismos aunque esto no implica que dichas estrategias sean leídas por la escuela como formas de participación de las familias en la escolarización de los hijos/as.

Por otra parte, si bien la escuela acepta y avala que los chicos/as asistan a centros de apoyo escolar, por lo general no se ponen ni solicitan ponerse en contacto con los referentes de estos centros para el seguimiento de la escola- 
Revista de la Escuela de Ciencias de la Educación, año 10, número 9, enero a diciembre de 2014. Páginas 197-212. ISSN 1851-6297. ISSN EN LINEA 2362-3349. "LAS FAMILIAS NO PARTICIPAN": UN ANÁLISIS SOBRE EL DESPLIEGUE DE SENTIDOS en la vida escolar. Carolina Scavino.

rización de los niños/as, en este punto siguen sosteniendo a las familias como los referentes escolares responsables.

Este desencuentro y falta de claridad en la delegación de las funciones entre familias y centros de apoyos y entre escuela y centros, termina finalmente recayendo sobre la "responsabilidad del niño/a" y da lugar a opiniones sobre la "educabilidad" de los mismos.

En la investigación sin embargo se han registrado casos aislados, como veremos a continuación, donde los centros comunitarios asumen la delegación plena de las familias y la escuela acepta de algún modo esta delegación permitiendo un intercambio fructífero para el proceso de escolarización de los niños/as.

\section{- "Las familias no participan" desde el punto de vista de los centros comunitarios con funciones educativas.}

A partir del trabajo realizado en la investigación sobre los centros comunitarios, se pudo registrar que la mayor parte de éstos reconocen a la familia como el interlocutor válido para interactuar con la escuela y consideran que sus servicios se limitan a prestar apoyo.

Si bien los destinatarios de su accionar son los niños/as, los motivos de su asistencia están en relación a las dificultades que presentan las familias de contextos vulnerables para ayudar a sus hijos/as en las tareas que demanda la escuela y no en relación a las dificultades que tiene la escuela para garantizar su objetivos. Planteárselo de este modo podría acercar el diálogo de las organizaciones de la comunidad con la escuela respecto a los problemas y/o dificultades que aparecen en los niños/as.

En varias de las entrevistas realizadas con referentes de los centros de apoyo escolar, se pudo registrar lo señalado anteriormente. Ellos consideran que "la escuela es de la mamá" justificando porque no adoptaban una postura más activa en el trabajo con el niño/a.

“(...) Estamos en contacto más que nada por ellos (...) yo a las maestras ni las conozco". Refiriéndose a un contacto de tipo informativo con la escuela de una vez por mes para ver si los chicos asisten o no al centro comunitario y/o a la escuela y en caso contrario comenzar con alguna otra tarea necesaria para garantizar la asistencia al establecimiento.

Sin embargo los centros de apoyo que avanzan en un contacto más fluido con la escuela, igualmente encuentran dificultades, "nosotras cuando hicimos (...) un recorrido por las escuelas (...) nos recibieron hasta ahí, no nos dieron mucha bolilla, (...) fui por los grados.... (...) fui golpeando la puerta, y entro y la maestra está dando la clase... fuiste (...) no te puede atender....". En esta entrevista se pone en evidencia que a pesar de adoptar una postura más activa, aparecen dificultades en los modos y formas de establecer los contactos así como en las posteriores instancias de trabajo. 
Revista de la Escuela de Ciencias de la Educación, año 10, número 9, enero a diciembre de 2014. Páginas 197-212. ISSN 1851-6297. ISSN EN LINEA 2362-3349. "LAS FAMILIAS NO PARTICIPAN": UN ANÁLISIS SOBRE EL DESPLIEGUE DE SENTIDOS en la Vida escolar. Carolina Scavino.

En algunos centros, las docentes responsables de trabajo diario en las tareas de apoyo, suelen realizar una visita inaugural a los establecimientos educativos a los que asisten sus chicos/as y luego queda como contacto una coordinadora como referente de articulación, que a su vez tiene a su cargo diferentes escuelas. Esta forma de organización también presenta dificultades para una articulación fluida y de referencia con la institución.

Sólo en uno de los centros estudiados, que trabaja en torno a la noción de "educación no formal", se observó un trabajo sistemático y consistente por articular con la escuela. Sobre esta experiencia comentaban: "con la escuela nos ha pasado varias cosas, también tiene que ver con la escuela ¿no?, una de las cosas que nos pasa es que hacer educación no formal parece que somos menos, que acá los chicos no hacen nada, entonces nos ha pasado eso como que no nos escuchan de la misma manera, y cuando sugerís cosas o planteas algo: "lo que pasa es que con ustedes se portan bien porque lo tienen todo el día jugando'. (...) También pasa -señalan en relación al tiempo que viene trabajando en el barrio- que hay maestras que te dicen ' $y$ y ustedes quien son?' $y$ hay que volver a contar porque no hay en la escuela una transmisión al interior del equipo..." Esta entrevista pone en evidencia ciertos obstáculos no solo de la dinámica escolar, como se pudo mostrar en otra de las citas anteriormente presentada, sino también respecto a la concepción y sentido de la educación que circula al interior de las escuelas. La resistencia de la escuela ante una concepción diferente en el modo de encarar el trabajo de acompañamiento en la escolaridad del niño/a.

\section{Reflexiones finales}

A partir de lo investigado se han puesto en evidencia ciertas tensiones que regulan el vínculo de la familia con la escuela así como la hegemonía de algunos sentidos sobre el significado de la frase "las familias no participan".

La frecuencia con la que se apela a la mencionada frase en el ámbito escolar para justificar las dificultades que tienen en el logro de sus objetivos, es comparable a las explicaciones del fracaso escolar por problemas individuales y/o socioculturales y familiares (Baquero, 2000, 2003); en ambos casos estos enunciados obstaculizan un análisis de la situación escolar que permitiría abrir nuevas alternativas frente a la diversidad que habita a las escuelas y el cumplimiento de los objetivos que persiguen.

A su vez, se ha observado que docentes como padres comparte el supuesto de que la escolarización y el rendimiento escolar, son productos de esfuerzos y capacidades individuales. De este modo, cuando la escuela reclama colaboración y apoyo ante a los problemas de escolarización del niño/a, y la familia acepta la demanda escolar e intenta resolver "en la medida de sus posibilidades"; la persistencia de los problemas redundará en el supuesto de que el "chico/a 
Revista de la Escuela de Ciencias de la Educación, año 10, número 9, enero a diciembre de 2014. Páginas 197-212. ISSN 1851-6297. ISSN EN LINEA 2362-3349. "LAS FAMILIAS NO PARTICIPAN": UN ANÁLISIS SOBRE EL DESPLIEGUE DE SENTIDOS en la vida escolar. Carolina Scavino.

es el problema" o bien, en el mejor de los casos, en un aumento del malestar de la escuela hacia lo que hace la familia y viceversa.

La mirada individualizada sobre el desempeño del alumno/a en la escuela también es compartida por gran parte de los centros socio-comunitarios que prestan "ayudas escolares", y focalizan su accionar en el "chico/a problema".

De este modo, los procesos de participación de la familia en la escuela no pueden ser analizados sin contemplar este sentido compartido respecto del responsable último de la escolarización: el niño/a.

En la investigación también se pudo dar cuenta que la "falta de participación de las familias" entra en contradicción si se observa la dinámica escolar. La escuela regula la circulación de las familias al interior de la institución, pauta las formas en que debe participarse, los momentos en que pueden suceder y tiene autoridad para definir los términos de la convocatoria. Las familias deben respetar y respetan este orden de cosas porque consideran a la escuela como un ámbito específico de formación para sus niños/as; pero también juzgan los pedidos/demandas que formula la escuela como válidas o no, en función de la especificidad y competencia que le reconocen, por ejemplo, cuando la escuela reclama sobre conductas o formas de actuar de los niños/as en vida diaria en el establecimiento, las familias siente que tiene poco o nada que hacer ya que al no estar presentes no pueden controlar a sus hijos/as.

Por otra parte, se observó que las familias hacen cosas que pueden no estar visualizadas en la escuela, por ejemplo enviar a sus hijos/as a los apoyos escolares y delegar en éstos la tarea de acompañamiento. Esta es una forma de participación bastante frecuente y poco reconocida por la escuela, no tanto por no estar informada sino porque siguen sosteniendo a la familia como el referente del acompañamiento, cuando éstas ya han cedido su responsabilidad a los centros de apoyo.

De un modo u otro, las formas de participación de la familia y la escuela respecto a las dificultades que se manifiestan en la escolarización de los niños/ as terminan situando a los pequeños/as como "objeto de intervención" más que agentes protagónicos en la resolución de los mismos.

Sólo en un caso de los estudiados, se pudo registrar un trato diferente con el "chico problema" que habilitó establecer una alternativa a los modelos tradicionales de participación entre la familia y la escuela.

El caso está en relación a un centro educativo que trabajó en el marco de la educación no formal y que pudo darle lugar a la palabra del niño respecto a sus problemas con la escuela. El chico les planteó que no podía asistir a natación porque su mamá no le había gestionado el certificado médico, fue entonces cuando los docentes del centro entraron en contacto con la escuela y la familia. La escuela reclamaba que la madre no se presentaba en el establecimiento al ser citada ni enviaba el certificado médico; la madre comentaba que como jefa de hogar y razones laborales, no podía presentarse en los horarios pautados 
Revista de la Escuela de Ciencias de la Educación, año 10, número 9, enero a diciembre de 2014. Páginas 197-212. ISSN 1851-6297. ISSN EN LINEA 2362-3349. "LAS FAMILIAS NO PARTICIPAN": UN ANÁLISIS SOBRE EL DESPLIEGUE DE SENTIDOS en la Vida escolar. Carolina Scavino.

por la escuela así como tampoco podía gestionar el certificado en cuestión. Los docentes del centro de apoyo comenzaron entonces a trabajar para que la escuela pueda comunicarle a ellos lo que necesitaba y que ellos asumirían la responsabilidad de gestionar lo necesario. Fue a partir de este modo alternativo de asumir el cuidado del niño, que el chico pudo retomar sus actividades cotidianas en la escuela.

En este sentido se considera que sostener el malestar que "las familias no participan" actúa como un obstáculo para avanzar en la comprensión de los problemas que aqueja a la escuela, en particular para visualizar caminos alternativos que podrían habilitarse para lograr una buena escolarización del alumno/a. La escuela al no habilitar a otros agentes socio-comunitarios así como al propio alumno/a, como interlocutores válidos de los problemas que se le presentan, restringe las posibilidades de su accionar. Por el contrario, aumenta su malestar, delegando y responsabilizando a las familias la imposibilidad de cumplir con sus metas escolares y reforzando incluso el supuesto de "déficit individual".

No pude dejarse de señalar, que la lógica con que se articulan estos procesos interactivos y los malestares a ellos asociados, encubre un juego de poder respecto a las responsabilidades y delegaciones entre los agentes sociales (familias y escuela) que termina produciendo efectos en los niños/as y sus trayectorias escolares. La escuela demanda a la familia, la familia recurre a los centros educativos comunitarios y éstos ubican nuevamente a la familia como el responsable de la escolaridad del niño/a. Este complejo accionar, pocas veces explicitado, no solo oscurece el campo de acción y la definición de objetivos concretos que permitan abordar el problema que la escuela reclama, sino que por su complejidad, genera condiciones de confusión en el niño/a respecto a lo que es necesario trabajar y muchas veces, ellos/as terminan convencidos que "el problema son ellos/as".

Finalmente cabe reflexionar sobre el "peso empírico" que porta la frase "las familias no participan". A lo largo del trabajo, se pudo dar cuenta que las formas de participación de las familias en la escuela están disueltas en la pluralidad y diversidad de un conjunto de acciones -entrevistas individuales, reuniones grupales de padres, mensajes particulares y generales a través del cuaderno de comunicados, asistencia a eventos escolares, etc- que son evaluadas en su individualidad, es decir en el número de familias que cumplen con las acciones que la escuela demanda. La escuela, a pesar de dirigirse a un grupo de padres, evalúa los casos en forma particular, individualiza a los chicos/as y sus familias, en el cumplimiento de las metas escolares, siempre se evalúa de modo personalizado. Es en esa operatoria de individuación la frase "las familias no participan" cobra su "peso empírico" en todas aquellas familias que no cumplen con lo establecido y esperado por la escuela y en el caso particular de aquellas instituciones que trabajan con población socioeconómicamente 
Revista de la Escuela de Ciencias de la Edducación, año 10, número 9, enero a diciembre de 2014. Páginas $197-212$. ISSN 1851-6297. ISSN EN LINEEA 2362-3349. "LAS FAMILIAS NO PARTICIPAN": UN ANÁLISIS SOBRE EL DESPLIEGUE DE SENTIDOS en la vida escolar. Carolina Scavino.

vulnerable, la frase de "no participación" encuentra más argumentos para señalar dificultades en los entornos escolares y mayores resistencias por parte de las instituciones educativas en asumir los problemas que tienen a partir del diseño de sus prácticas y formas de organización en el cumplimiento de sus objetivos que se proponen.

\section{Notas bibliográficas}

(1) Este estudio se desarrolló en el marco de un proyecto de Investigación y Desarrollo de la Universidad Nacional de Quilmes bajo la dirección de la Dra. Ma. Cristina Chardon titulado: "Instituciones y sujetos del cuidado. Transformaciones actuales de las representaciones y prácticas en el ámbito de la salud, la educación y las familias". En este proyecto las prácticas familiares son señaladas como ámbitos que definen un "aprendizaje sobre el cuidado" sobre los integrantes que la componen y que posteriormente, éstos pueden complementar y/o revisar dichos aprendizajes en el tránsito por otras prácticas como por ejemplo la escuela. De este modo, también puede advertirse el carácter creador que define a las prácticas familiares en contraposición a las escolares: "(...) los intercambios ciudadanos construyen -y se construyen en- concepciones de cuidado que se forjan en prácticas colectivas en las que se reconstruyen las nociones de cuidado inauguradas, producidas y reproducidas en los ámbitos domésticos de cuidado" (Chardon, et al, 2011: 270).

(2) El estudio de caso se desarrollo en el marco de un proyecto de investigación para el Programa de Doctorado con Mención en Cs. Sociales y Humanas de la Universidad Nacional de Quilmes. El título de la tesis (inédita) es: "Las familias en la escuela: procesos de participación y comunidad de aprendizaje en contextos de pobreza urbana".

(3) Al referir a "intereses" se está recuperando la noción de Illusion de Bourdieu quien considera que este término debe diferenciarse de la noción idealista y del sentido común, tradicionalmente conocida como "motivo". Para el autor los intereses son los modos en que los agentes sociales se mueven en un campo y le dan funcionamiento a medida que juegan el juego, invierten en el juego y sus reglas.

(4) En la tesis doctoral se pudo rastrear que las relaciones entre la familia y la escuela que actualmente eran vistas de modo negativo, no eran percibidas de la misma forma en la reconstrucción histórica del establecimiento. El cambio en las formas de percibir el vínculo estaba asociado a transformaciones socio-comunitarias que afectaron a la organización de la escuela como por ejemplo el cambio de jornada doble a simple con el objetivo de ampliar la cobertura escolar de los niños/as de la zona (Scavino, 2012).

(5) La expresión "deseada" no solo quiere indicar el anhelo de algo sino también el carácter mítico que adquiere la frase en el discurso escolar.

(6) Para ampliar sobre las condiciones de trabajo en la que se desarrolla la tarea docente en contextos de vulnerabilidad social, se sugiere ver ACIJ-Junco Film (2008) "El sur también existe", Documental sobre la desigualdad educativa en la Ciudad de Buenos Aires, Buenos Aires, Argentina. Recuperado de: http://vimeo.com/1223051

(7) Esta observación es pertinente para la población socioeconómicamente vulnerada con la que se trabajo en este estudio. 
Revista de la Escuela de Ciencias de la Educación, año 10, número 9, enero a diciembre de 2014. Páginas 197-212. ISSN 1851-6297. ISSN EN LINEA 2362-3349. "LAS FAMILIAS NO PARTICIPAN": UN ANÁLISIS SOBRE EL DESPLIEGUE DE SENTIDOS en la Vida escolar. Carolina Scavino.

\section{Referencias Bibliográficas}

- Achilli, E. (2010). Escuela, Familia y desigualdad social. Argentina: Laborde.

- (et. Al). (2000). Escuela y Ciudad. Exploraciones de la vida urbana. Argentina: Universidad Nacional de Rosario Editora.

- Baquero, R. (2000). Lo habitual del fracaso, el fracaso de lo habitual. En F. C. Avendaño y N. Boggino (Comps.) La escuela por dentro y el aprendizaje escolar. Rosario: Homo-Sapiens.

- Bourdieu, P. (2007). Crítica de la razón Práctica. En El sentido práctico. Buenos Aires: Siglo XXI.

- $\quad$ Bravin, C. (2004). Escuelas, familias y mujeres. Argentina: Novedades Educativas.

- Bruner, J. (2013). La fábrica de historias. Argentina: Fondo de Cultura Económica.

- Cárcova, C. M. (1998). La opacidad del Derecho. Madrid: Trotta.

- Cerletti, L. (2012). Familias y Participación: un análisis comparativo de la Ley 1420, la Ley Federal de Educación y la Ley de Educación Nacional. En Propuesta Educativa n 37, Junio 2012.

$\cdot$ (2010). Familias y Escuelas: aportes de una investigación etnográfica a la problematización de supuestos en torno a las condiciones de escolarización infantil y la categoría "familia". En Intersecciones en Antropología. 11 (1), Junio 2010.

(2008). Tras los sentidos de "participación": un análisis desde la etnografía educativa. En Avá-Revista de Antropología, $n^{\circ}$ 13, Julio 2008.

$\cdot$ cativas. (2006). Las Familias, ¿un problema escolar? Argentina: Novedades Edu-

- Chardon, M. C. (2011). Introducción. En M. C. Chardón (Coord.) Transformaciones del Espacio Público. Buenos Aires: La Crujía.

- Chardon, M. C., Resemar, S., Cacciavillani, F., Leegstra, R. y Cristiani, L. (2011a). Cuidado y actividades de la vida diaria. En M. C. Chardon (Coord.) Transformaciones del Espacio Público. Buenos Aires: La Crujía.

- $\quad$ Chardon, M. C., Borakievich, S., Ferreyra, M., Pegoraro, V. (2011 b). La construcción del cuidado y la alteridad. En M. C. Chardon (Coord.) Transformaciones del Espacio Público. Buenos Aires: La Crujía.

- Douglas, M. (1996). Cómo piensan las instituciones. España: Alianza.

- Duranti, A. (2000). Antropología Lingüística. España: Akal.

- Herzfeld, M. (1992). The social production of indifference: exploring the symbolic roots of western bureacracy. USA: University of Chicago Press.

- Jodelet, D. (2006). El otro, su construcción, su conocimiento. En S. Valencia Abundiz (Coord.) Representaciones Sociales: Alteridad, Epistemología y Movimientos Sociales. México: Universidad de Guadalajara.

- _ (1988). La representación social: fenómenos, conceptos y teoría. En S. Moscovici (Comp.) Psicología Social II. Barcelona: Ed. Paidos.

- Jovchelovitch, S. (2007). Knowledge in context: Representations, Community and Culture. Canadá: Routledge.

- Markova, I. (2006). Sobre las formas de interacción del reconocimiento social. En S. Valencia Abundiz (Coord.) Representaciones sociales: alteridad, Epistemología y Movimientos Sociales. México: Universidad de Guadalajara.

- Neufeld, M. R. (2000). Familias y Escuelas: la perspectiva de la antropología social. Ensayos y Experiencias, $n^{\circ} 36$.

- Rockwell, E. (2010). Tres planos para el estudio de las culturas escolares. En N. Elichiry (Comp.) Aprendizaje y Contexto: Contribuciones para un debate. Buenos Aires: Manantial. 
Revista de la Escuela de Ciencias de la Educación, año 10, número 9, enero a diciembre de 2014. Páginas 197-212. ISSN 1851-6297. ISSN EN LÍNEA 2362-3349. "LAS FAMILIAS NO PARTICIPAN": UN ANÁLISIS SOBRE EL DESPLIEGUE DE SENTIDOS en LA VIDA ESCOLAR. CaRolina ScaVino.

- Santillan, L. (2008a). Las Familias en la escuela. En Serie Explora Pedagogía, Ministerio de Educación de la Nación. Recuperado de: http://explora.educ.ar/wp-content/ uploads/2010/04/PEDAG06-Las-familias-en-la-escuela.pdf

- $\quad$ (2008b). Trayectorias educativas y cotidianidad: una etnografía del problema de la educación y la experiencia escolar en contextos de desigualdad. Propuesta Educativa. $\mathrm{n}^{\circ} 30$, noviembre 2008.

- _ (2007). La "educación" y la "escolarización" infantil en tramas de intervención local. Una etnografía en los contornos de la escuela. Revista Mexicana de Investigación Educativa, 12(34).

(2006). La construcción social del problema de la educación: un estudio antropológico desde la perspectiva y los modos de vida de los grupos familiares. Intersecciones en Antropología, n7, enero-diciembre 2006.

- $\quad$ Santillan, L. y Cerletti, L. (2011). Familias y escuelas: repensando la relación desde el campo de la Antropología y la Educación. Boletín de Antropología y Educación, Año 2, $\mathrm{n}^{\circ} 3$, diciembre 2011.

- Scavino, C. (2012). Las familias en la escuela: procesos de participación y comunidad de aprendizaje en contextos de pobreza urbana (Tesis inédita de Doctorado). Universidad Nacional de Quilmes. Bernal, Buenos Aires, Argentina.

(2011). Estudio sobre procesos de participación familia y escuela: reflexiones sobre un esquema conceptual de análisis. En Jornada de Becarios del Departamento de Ciencias Sociales, Bernal, Buenos Aires, Argentina. Universidad Nacional de Quilmes.

(2007). Prácticas de crianza y Consulta de Control de Niño Sano: un análisis sobre intervenciones en salud en contextos de pobreza (Tesis inédita de Maestria). Facultad de Psicología, UBA. Buenos Aires, Argentina. 